\title{
Correspondence
}

\section{Pseudofolliculitis barbae induced by oral minoxidil}

doi: 10.1111/j.1365-2230.2012.04376.x

Oral minoxidil, a drug used to treat severe hypertension, can have effects on hair growth. We report a patient with pseudofolliculitis barbae induced by oral minoxidil.

A 61-year-old Afro-Caribbean man presented with a 5year history of tender and inflamed papules and pustules, associated with keloid scarring in the beard area extending onto his cheeks and posterior scalp. His medical history included hypertension, glaucoma and gout, for which he was being treated with amlodipine, oral minoxidil $100 \mathrm{mg}$ daily, doxazocin and allopurinol.

On physical examination, ingrowing hairs and large pustules were evident in the patient's beard area (Fig. 1a,b).

Microbiology swabs and tissue samples grew Proteus mirabilis, Enterococcus faecalis and mixed anaerobes. On histological examination of a biopsy taken from the affected area, an intrafollicular and perifollicular multifocal chronic inflammatory infiltrate composed mainly of lymphocytes, plasma cells and neutrophils was identified These inflammatory infiltrates had destroyed the hair follicles, causing an adjacent giant cell-reaction and marked fibrosis (Fig. 2).

A clinical diagnosis of pseudofolliculitis barbae and acne keloidalis nuchae was made. The patient was initially treated with topical antiseptics, topical erythromycin with moderate-strength topical steroid, intralesional triamcinolone, oral erythromycin, oral vibramycin and oral isotretinoin, which provided temporary relief. However, because of the severity of the condition and its recalcitrant nature despite medical treatment, surgical removal and destruction (electrodessication and debulking) of the abnormal tissue was undertaken. The surgical wounds healed well with no keloidal scarring, but the pseudofolliculitis persisted. However, dramatic improvement was noted after cessation of the patient's oral minoxidil (Fig. 1c,d), with the erythema, oedema and pustular exudates reducing to reveal quiescent skin in the affected areas within 6 weeks.

In retrospect, when the patient had started oral minoxidil 15 years previously, he had noticed a generalized increased in hair growth, but the pseudofolliculitis did not develop for 10 years.
Figure $1(a, b)$ Large proliferative plaque of keloids on (a) the right and (b) the left side of the face; (c,d) significant clinical improvement after surgical treatment and cessation of oral minoxidil.
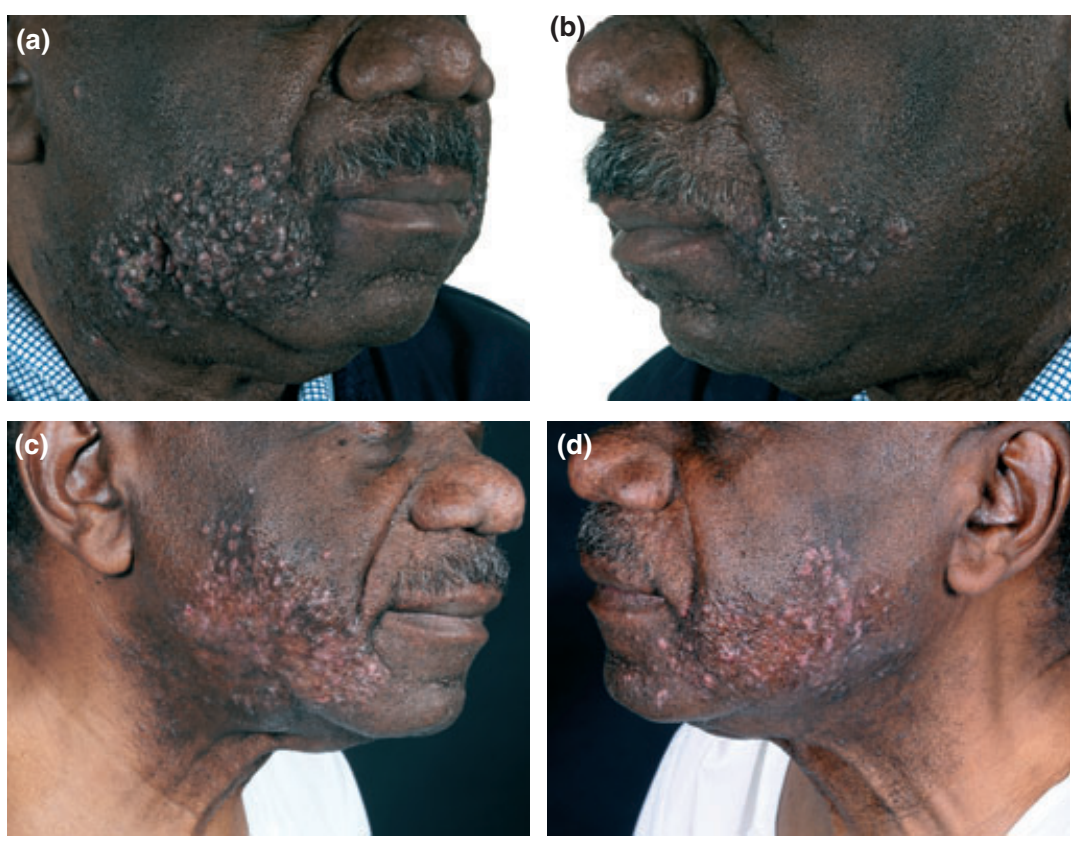


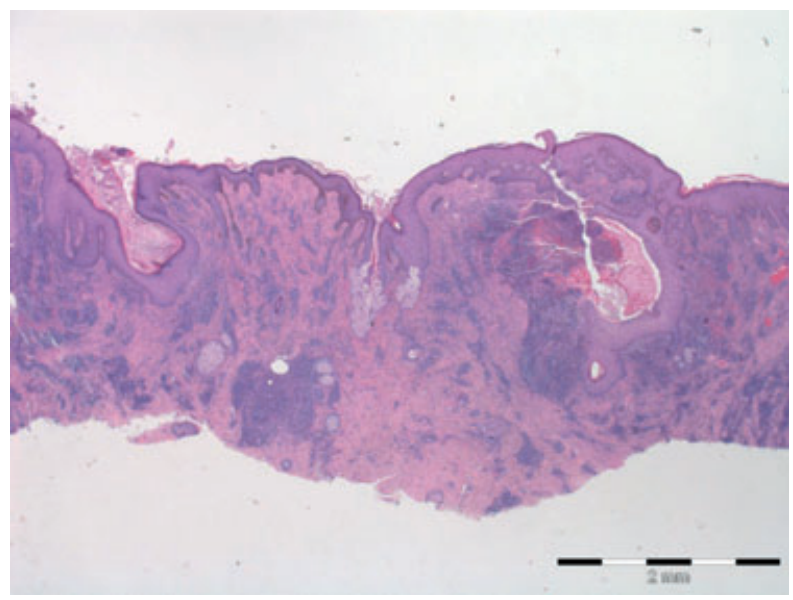

Figure 2 Chronic inflammatory infiltrate with evidence of ruptured abscess and fibrosis (haematoxylin and eosin, original magnification $\times 12.5$ ).

Oral minoxidil was originally developed to treat hypertension, but was found to produce hypertrichosis in 59\% of cases, ${ }^{1}$ which subsequently led to the development and use of a topical formulation for male-pattern baldness.

The precise mechanism underlying the effect of oral minoxidil on hair growth remains unclear, although a number of mechanisms have been proposed, including local vasodilatation, angiogenesis, ${ }^{1}$ immunosuppression, suppression of collagen synthesis, and enhanced cell proliferation and DNA synthesis in follicular and perifollicular cells. ${ }^{2}$ On histological examination, the anagen phase of hair growth is seen to be prolonged, and the action of minoxidil is at the dermal papillae, ${ }^{3,4}$ where increased expression of vascular growth endothelial factor mRNA occurs ${ }^{5}$. We suspect that these stimulatory changes induced by oral minoxidil may have contributed to the development and severity of the pseudofolliculitis and acne keloidalis in our patient, gradually worsening over the years.

Withdrawal of minoxidil reverses the proposed mechanism of actions on the follicular unit. Concurrent with the improvement of the folliculitis, our patient noticed a decrease in hair on his scalp. The reversal of the clinical condition adds weight to the argument that his symptoms were drug-induced.

In conclusion, we believe our patient's skin condition was pseudofolliculitis barbae and acne keloidalis nuchae, triggered by the long-term use of oral minoxidil, the first report of such an association to our knowledge.

\section{H. M. Liew, R. Morris-Jones, S. Diaz-Cano and S. Bashir} Department of Dermatology, King's College Hospital, London, SE5 9RS, UK

E-mail:h.liew@nhs.net

Conflict of interest: none declared.

Accepted for publication 27 November 2011

\section{References}

1 Burton JL, Marshall A. Hypertrichosis due to minoxidil. Br J Dermatol 1979; 101: 593-5.

2 Rogers NE, Avram MR. Medical treatments for male and female pattern hair loss. J Am Acad Dermtol 2008; 59: 567-8.

3 Headington JT, Novak E. Clinical and histologic studies of male pattern baldness treated with topical minoxidil. Curr Ther Res 1984; 36: 1098-106.

4 Headington JT. Hair follicle biology and topical minoxidil: possible mechanism of action. Dermatologica 1987; 175 (Suppl. 2): 19-22.

5 Lachgar S, Charveron M, Gall Y et al. Minoxidil upregulates the expression of vascular endothelial growth factor in human hair demal papilla cells. Br J Dermatol 1998; 138: 407-11. 